\title{
Endovascular Coil Embolization of Very Small Intracranial Aneurysms
}

\author{
Kil Sung Chae, $\mathrm{MD}^{1,2}$ \\ Pyoung Jeon, MD ${ }^{1}$ \\ Keon Ha Kim, MD' \\ Sung Tae Kim, MD' \\ Hyung Jin Kim, MD ${ }^{1}$ \\ Hong Sik Byun, MD
}

\section{Index terms : \\ Intracranial aneurysm \\ Endovascular treatment \\ Outcome}

DOI:10.3348/kjr.2010.11.5.536

Korean J Radiol 2010; $11: 536-541$

Received March 23, 2010; accepted

after revision June 16, 2010.

'Department of Radiology and Center for Imaging Science, Samsung Medical

Center, Sungkyunkwan University School of Medicine, Seoul 135-710, Korea;

${ }^{2}$ Department of Neurosurgery, Bong Seng

Memorial Hospital, Busan 601-723, Korea

Corresponding author:

Pyoung Jeon, MD, Department of

Radiology, Samsung Medical Center,

Sungkyunkwan University School of

Medicine, 50 llwon-dong, Gangnam-gu,

Seoul 135-710, Korea.

Tel. (822) 3410-2518

Fax. (822) 3410-2559

e-mail: drpjeon@gmail.com
Objective: We aimed to evaluate the results of endovascular coil embolization for very small aneurysms ( $\leq 3 \mathrm{~mm}$ ).

Materials and Methods: Between March 2005 and December 2008, a total of 31 very small aneurysms in 30 patients were treated by coil embolization. Of the 31 aneurysms, five (16\%) were ruptured, as opposed to 26 (84\%) that were not. We assessed the procedural complications, immediate angiographic outcome after coiling, clinical outcome, and follow-up MR angiography (MRA).

Results: Two thromboembolic complications occurred during the procedure, but did not lead to any persistent neurologic deficit. No procedural aneurysmal rupture was observed and procedure-related morbidity and mortality were both $0 \%$. Occlusion was adequate in 25 aneurysms (81\%) and incomplete in six aneurysms (19\%). The clinical outcomes of five patients with ruptured aneurysms were good (Glasgow outcome scale $\geq 4$ ), with no bleeding of the treated aneurysms during a mean follow-up period of 13.3 months. On 27 follow-up MRA, there was no recurrence, and the five incompletely occluded aneurysms showed a spontaneous amelioration resulting in an adequate occlusion.

Conclusion: Coil embolization of very small aneurysms is technically feasible with good results. The long-term efficacy and the potential as a standard treatment strategy remain to be determined by randomized large trials.

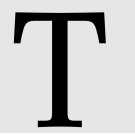

he International Subarachnoid Aneurysm Trial acknowledged the greater efficacy of endovascular treatment of ruptured aneurysms compared to clipping. But in this trial, very small aneurysms $(\leq 3 \mathrm{~mm})$ were not included, and the conclusion that coiling is the preferred treatment does not apply to these very small aneurysms (1).

Endovascular treatment of very small aneurysms is considered to be technically challenging, and high complication rates have been reported $(2,3)$. The small size of the aneurysmal sac, in these cases, restricts to obtain a stable microcatheter position and coil detachment. As a result, this increases the risk of procedural rupture $(2,4)$. However, with the advent of more sophisticated endovascular materials, the growing experience of interventionists, and an increased use in adjunctive techniques, such as balloon or stent assisted coiling, several studies have shown that endovascular treatment of very small aneurysms can be done with acceptable risk $(2,3,5-7)$.

Nonetheless, the efficacy of endovascular treatment in very small aneurysms and the management of unruptured, very small aneurysms are unclear, and remain under continued investigation.

Thus, we retrospectively reviewed our consecutive, small series to evaluate the outcome of endovascular coil embolization in very small aneurysms including a large 
proportion of unruptured aneurysms.

\section{MATERIALS AND METHODS}

\section{Patients}

Between March 2005 and December 2008, a total of 810 aneurysms were treated by either coil embolization or surgery at our institution. Of the 468 aneurysms treated by surgery, $142(30 \%)$ were very small $(\leq 3 \mathrm{~mm})$ and of these, $40(28 \%)$ were ruptured aneurysms. Whereas, of the 342 aneurysms treated by coil embolization, 31 (9\%) in 30 patients were very small and five of them were ruptured (16\%). Thus, we enrolled these 31 very small aneurysms from the 30 patients for this study. The 30 patients consisted of 11 males (37\%) and 19 females $(63 \%)$ with a mean age of 51.1 years (median, 52 years; range, 28-81 years).

The Hunt and Hess (HH) Grade of the five patients who had presented ruptured aneurysms before endovascular treatment included one Grade IV, two Grade II, and two Grade I patients. The other 26 very small aneurysms that were not ruptured were incidentally diagnosed during imaging studies obtained for various other clinical reasons.

The locations of the 31 aneurysms included $16(52 \%)$ in the internal carotid artery, four $(13 \%)$ in the anterior communicating artery, four $(13 \%)$ in the posterior communicating artery, two $(7 \%)$ in the internal carotid artery (ICA) bifurcation, two $(7 \%)$ in the superior cerebellar artery, one (3\%) in the anterior choroidal artery, one $(3 \%)$ in the middle cerebral artery, and one $(3 \%)$ in the basilar tip. The aneurysm locations of this series are summarized in Table 1.

\section{Endovascular Coil Embolization}

All endovascular procedures were performed on biplane angiographic systems (Alulla FD 20/10; Philips Medical Systems, Best, The Netherlands, Integris LCN+; GE, Milwaukee, WI). Rotational angiography, followed by three-dimensional (3D) reconstruction by volume rendering was performed before the procedure in all cases. Aneurysm size was determined as the maximal diameter on 3D angiography. Coil embolization was performed under general anesthesia. In unruptured aneurysms, 3,000-4,000 units of heparin were administered at the beginning of the procedure, followed by an additional 1,000 units per hour. Heparin was continued intravenously for 24 hours after embolization.

Several types of coils were used: Guglielmi detachable coils (GDC; Boston Scientific, Fremont, CA), Trufill-DCS (Cordis, Miami, FL), Microplex (Microvention, Aliso Viejo, CA), and Axium (ev3, Irvine, CA).
Seven $(23 \%)$ wide-neck aneurysms were coiled by using various neck-remodeling techniques. Two wide-neck aneurysms were treated with balloon assistance by HyperForm or HyperGlide (ev3). Four wide-neck aneurysms were coiled with stent assistance by Neuroform (Boston Scientific) and one wide-neck aneurysm was coiled with a combination of balloon and stent.

\section{Angiographic Outcome and Complications}

The immediate angiographic outcome after coiling was classified as an adequate or incomplete aneurysm. An adequate occlusion was defined as no contrast filling in the aneurysmal sac, no matter if there was neck remnant at the end of the procedure. Occlusion was considered as incomplete when contrast filling persisted in the remnant sac.

\section{Clinical and MR Angiographic Follow Up}

Patients treated by coil embolization were usually scheduled for a follow-up visit at the outpatient clinic at 36 weeks after coiling. Follow-up imaging was performed by 3D time-of-flight (TOF) MRA at 6 months, 1 year, 2 years, and 3 years. Some patients underwent a MRA the day the embolization to obtain a baseline. Clinical and MRA follow-up periods varied from six to 39 months and averaged 13.6 months.

Neurologic status according to the Glasgow Outcome Scale (GOS) was evaluated at six months and was classified as independent (GOS 4 and 5), dependent (GOS 2 and 3), and death (GOS 1). The 3D TOF-MRA was obtained on a 3.0T Philips Achieva system (Philips Medical systems) with the following 3D parameters: repetition/echo time (TR/TE) $25 / 3.5 \mathrm{~ms}$; flip angle $25^{\circ}$; matrix $880 \times 450$ pixels (voxel size $0.6 \times 0.24 \times 0.24 \mathrm{~mm}$ ); field of view (FOV) $250 \mathrm{~cm}^{2}$. Post-processing consisted of maximum intensity projections (MIPs) for $360^{\circ}$ around the head in both a left-to-

Table 1. Aneurysm Location

\begin{tabular}{lc}
\hline Location & Total, No. (\%) (Ruptured, No.) \\
\hline ICA (paraclinoid) & $16(52 \%)$ \\
ICA bifurcation & $2(7 \%)$ \\
AcoA & $4(13 \%)(3)$ \\
AchA & $1(3 \%)(1)$ \\
MCA & $1(3 \%)$ \\
PcoA & $4(13 \%)(1)$ \\
BA tip & $1(3 \%)$ \\
SCA & $2(7 \%)$ \\
\hline
\end{tabular}

Note. $-\mathrm{Ach} \mathrm{A}=$ anterior choroidal artery, $\mathrm{AcO} \mathrm{A}=$ anterior communicating artery, $\mathrm{BA}=$ basilar artery, ICA = internal carotid artery, $\mathrm{MCA}=$ middle cerebral artery, No. = number, $\mathrm{PcoA}=$ posterior communicating artery, SCA $=$ superior cerebellar artery 
right and head-to-foot rotation. Aneurysm outcome was classified as same as the DSA. An aneurysm was considered to be recurrent when a previously adequately occluded aneurysm was found to have partial or even less recanalization of the neck and sac.

\section{RESULTS}

\section{Angiographic Outcome}

Immediate aneurysmal occlusion was adequate in 25

(81\%) aneurysms (5 out of 5 ruptured aneurysms, 100\%;
20 out of 26 unruptured aneurysms, $77 \%$ ), and incomplete in six $(19 \%)$ aneurysms. For the six aneurysms with an incomplete occlusion, additional treatments were not performed because all six aneurysms were unruptured and the remnants of aneurysmal sac were not large enough to require additional retreatment. The count of used coils ranged from 1 to 5 (mean count, 2.4) and the mean packing density was $34 \%$.

\section{Procedural Complications}

Two thromboembolic complications (7\%) occurred

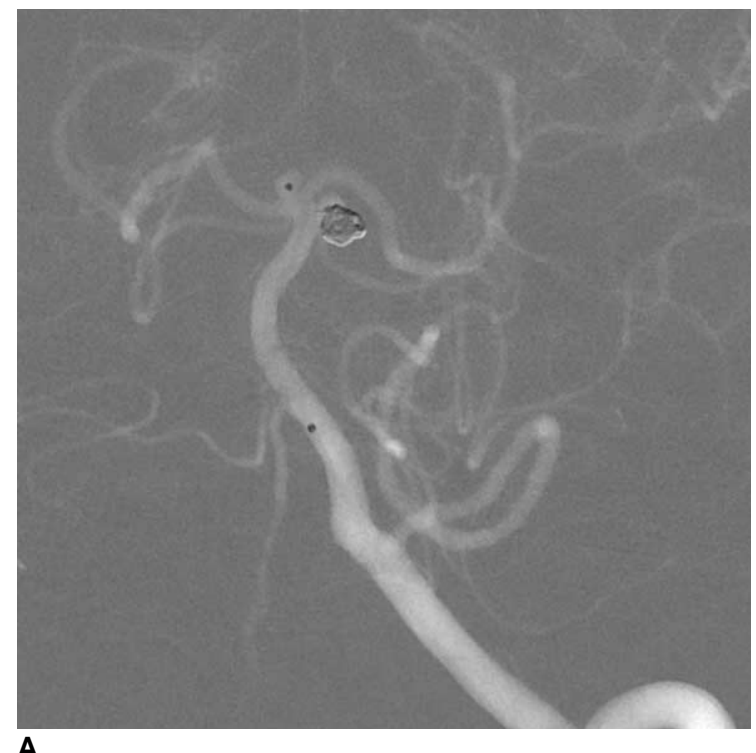

A

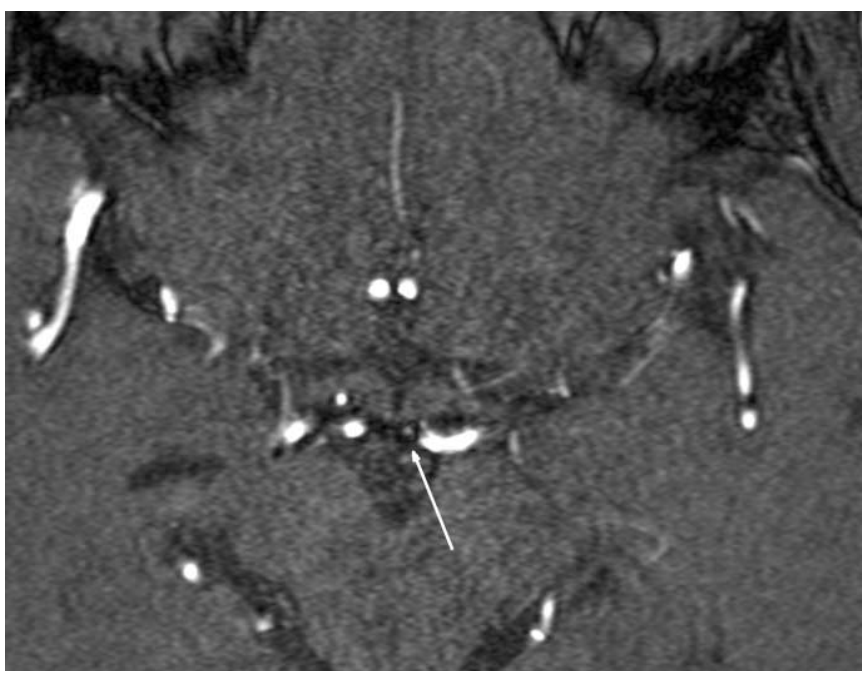

C

Fig. 1. Follow-up MR angiographic images in ages in 61-year-old woman.

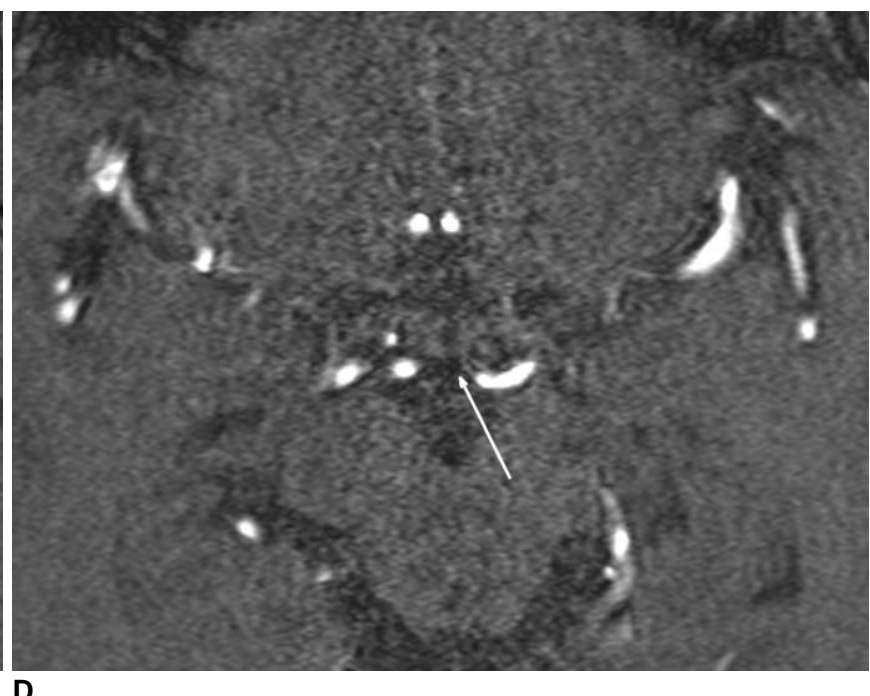

D

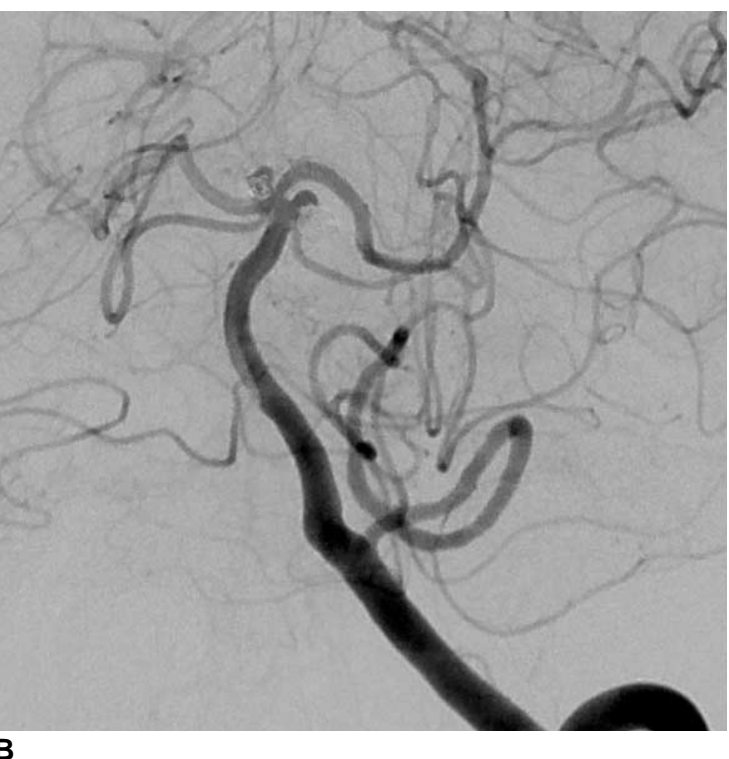

B

A. Roadmap image shows that microcatheter tip is in aneurysm on top of basilar artery. Another small aneurysm is noted between basilar artery and left superior cerebellar artery.

B. Postembolization angiography shows contrast filling of residual aneurysmal sac, especially in dome of aneurysm.

C. Source image of 3D TOF MRA on day following coil embolization depicts focal high signal intensity, thus suggesting residual arterial

flow in embolized aneurysm (arrow).

D. Source image of six month follow-up 3D TOF MRA shows loss of high signal intensity in embolized aneurysm (arrow). 


\section{Endovascular Coil Embolization of Very Small Intracranial Aneurysms}

during the procedure in two patients. One was a small thrombus in the parent artery initiating from the aneurysmal sac and the other was an in-stent thrombosis in the wide-neck aneurysm. Intra-arterial thrombolysis using abciximab $\left(\right.$ ReoPro $\left.{ }^{\circledR}\right)$, was successfully performed and the two patients had no permanent neurologic deficits. No procedural aneurysmal ruptures occurred nor procedurerelated complications leading to morbidity and mortality.

\section{Clinical Outcome}

Of the 31 aneurysms, a clinical follow-up was available in 30 aneurysms. Two patients who had presented in poor $\mathrm{HH}$ grade (IV) and good $\mathrm{HH}$ grade (II), respectively, had permanent focal neurologic deficit caused by subarachnoid hemorrhage, but both cases were independent (both, GOS 4) at six months follow-up. The other 27 patients had a good clinical outcome (GOS 5) with no bleeding of coiled aneurysms in all patients during the follow-up period.

\section{Follow Up MR Angiographic Results}

Twenty seven (87\%) of 31 aneurysms had follow-up MRA. Of the six incompletely occluded aneurysms on immediate angiography, five were followed. On MRA of these five aneurysms, focal high signal intensities representing remnant arterial flow within the coiled mass were absent or disappeared after serial follow-up (Fig. 1). No other recurrence was seen in the other 22 aneurysms.

\section{DISCUSSION}

In our study, endovascular coil embolization of very small aneurysms was technically feasible and showed successful results without procedural morbidity and mortality, although there were two thromboembolic events during the procedure not resulting neurologic deficits.

Coil embolization of very small aneurysms is considered to be technically challenging. Small aneurysms tend to have a thin fragile wall as well as restricted space for insertion of microcatheter and anchoring coils, and have been reported to have higher rates of procedure-related rupture. In a meta-analysis by Brinjikji et al. (8), the incidence of procedural rupture rate among the published studies was $8 \%$. No such procedural ruptures were observed in our series. In most cases, we used the microcatheter that had a shorter distal radiolucent portion over the distal marker and softer microwire (4). We tried a microwire that was never introduced into the aneurysm, and the microcatheter was shaped such that it pointed toward the dome of the aneurysm. The tip of the microcatheter was kept at the neck of the aneurysm as possible, and if necessary, the microcatheter was navigated farther into the aneurysm over the coil loop. The coil insertion was done very slowly, and delicate microcatheter manipulation was used to avoid tension build-up in the aneurysm and simultaneously allow the coil loop to form inside the aneurysm. The high magnification view was useful to observe the movement of coils and microcatheter tip inside the aneurysm. After the end of coil placement, the microcatheter was slowly withdrawn with microwire to minimize the force applied to the coil loops in the aneurysm. Even if minimal contrast filling was seen, no excessive additional coil placement was attempted.

A ruptured aneurysm was known as a risk factor for procedure-related rupture (9). Brinjikji et al. (8) confirmed that the incidence of procedural rupture was higher as $10 \%$ in ruptured very small aneurysms, compared to $5 \%$ in unruptured aneurysms. In the study by van Rooij et al. (5), investigating 47 unruptured and 149 ruptured very small aneurysms, procedural rupture occurred only in ruptured aneurysms. Our series had a large proportion of unruptured aneurysms (84\%), unlike most reports which enrolled patients with recently ruptured very small aneurysms. And our results are consistent with previous findings.

The most frequent location of very small aneurysms in our study was the internal carotid artery $(52 \%)$ in the paraclinoid region. In a large, single-center study, the location was significantly more common in the anterior cerebral artery and less common in the internal carotid artery (5). However, it included ruptured aneurysms in $76 \%$ of cases and did not demonstrate the data on the basis of ruptured or unruptured aneurysms. Two studies ( 7 , 8 ), one of which enrolled only unruptured aneurysms, whereas the other demonstrated the data of unruptured aneurysms separately, showed that the location of paraclinoid internal carotid artery was common $(41 \%$ and $43 \%$, respectively). In addition, all aneurysms with the location of paraclinoidal ICA were unruptured cases in our series. To our knowledge, there was no reference, including a large number of patients where the most frequent location was the ICA of the paraclinoid region in unruptured asymptomatic aneurysms. The Analysis of Treatment by Endovascular approach of Nonruptured Aneurysms (ATENA) study mentioned that the rate of procedural rupture was lower in ICA aneurysms than others $(2 \%$ in ICA, $2 \%$ in anterior cerebral artery/anterior communicating artery, $4 \%$ in MCA and $0 \%$ in posterior circulation) in the absence of statistical verification (8).

Incompletely occluded aneurysms (19\%) after coil embolizations were common in our series, which was not the case in previous reports (5\% in both van Rooij et al. [5] 


\section{Chae et al.}

and Brinjikji et al. [8]). The meta-analysis of Brinjikji et al. (8) reported that incomplete occlusion rates for ruptured and unruptured aneurysms were similar (8). However, the ATENA study investigating a large series of unruptured intracranial aneurysm less than $15 \mathrm{~mm}$, which was treated by the endovascular approach, showed a high incomplete occlusion rate $(19 \%)$ and relatively low procedural rupture rate $(3 \%)$, though there was no specification about the incomplete occlusion rates according to the size of aneurysms (7). Because our incompletely occluded aneurysms were unruptured, we might focus on the idea that aneurysm perforation during endovascular procedures should not occur and it might be the cause of not achieving a complete occlusion. Interventionist's vigorous tries for complete occlusion may actually increase the chance of procedure-related rupture. Consequently, a high rate of incomplete occlusion in our series may be relevant with regards to the absence of procedural rupture.

Of two thromboembolic complications, one $(50 \%, 1$ of 2 ) occurred in a patient with wide-neck aneurysm that was treated a remodeling technique using stent assistance. Balloon or stent assistance for the treatment of widenecked and complex lesions has become a routine adjunct to endovascular treatment of intracranial aneurysms in some groups (10). However, interventionists should be reminded that additional endovascular manipulations during the insertion of a balloon or stent may increase procedure-related complications.

The rate of aneurysm recurrence after coiling has been provided in various single and multicenter studies and has ranged from $5 \%$ to $33 \%$ (11-15). According to few reports investigating endovascular results in ruptured and unruptured very small aneurysms, the recurrence rate was $2-5 \%(5,7,8)$. In our series, none of the 27 aneurysms that were followed showed any recurrence. Nguyen et al. (16) reported that ruptured aneurysms and larger aneurysms were significantly associated with recanalization. Our experience and the previous few reports corroborate this.

Many paraclinoid ICA aneurysms were noted in our series, which were so-called side wall aneurysms, unlike other aneurysms of the bifurcation site. This type of aneurysms may be less influenced by hemodynamic forces including turbulent flow or direct jet pushing on the coiled mass, and as a result, may lead to the absence of recanalization.

An initial incomplete occlusion or loose packing of the aneurysm was significantly associated with recanalization (16). For aneurysms that are not tightly packed, coiled masses are exposed to the 'water-hammer effect,' which is responsible for aneurysmal regrowth. Therefore, achieving complete or near complete occlusion is the key to preventing recurrent hemorrhage and regrowth of the aneurysm when coiling aneurysms. However, it is quite interesting that our five incompletely occluded aneurysms that were followed by MRA showed focal high signal intensities within the coiled aneurysmal sac, which represented arterial flow that disappeared on serial follow-up. The results suggest that even if occlusion is incomplete on immediate angiography after coil embolization, adequate occlusion may eventually be achieved. Though it is difficult to know what may progress to an adequate occlusion in these cases. We could suppose that the inflow zone can be more easily occluded in very small aneurysms because of the absolute small size of the inflow zone compared to larger aneurysms. However, our series is too small and has too short of a follow-up period; therefore, further studies with larger trials and longer follow-ups should be accumulated to ensure the course of incompletely occluded, unruptured, very small aneurysms.

Our mean packing density was $34 \%$. The incidence of recanalization was significantly lower in aneurysms with coil packing densities $>25 \%$ (12). The recurrence rate of endovascular treatment for very small aneurysms remains to be determined through additional studies.

Very small aneurysms were common at our institution. They comprised $9 \%$ of all coiled aneurysms and consisted of up to $30 \%$ of surgically treated aneurysms. Moreover, incidental very small aneurysms are more frequently detected with the development of neuroimaging and the increase of availability. The results of the International Study of Unruptured Intracranial Aneurysms (ISUIA) suggested that small unruptured aneurysms have a benign natural course and a very low risk of bleeding when left untreated (17). There is no strict standard as to whether to treat or how to treat unruptured very small aneurysms in our institute. Each patient is evaluated in a joint meeting of neurosurgeons and interventional neuroradiologists. The decision to treat very small aneurysm is made on a caseby-case basis taking into consideration the following facts: 1) previous history of subarachnoid hemorrhage from another location, 2) presence of a family history of subarachnoid aneurysmal hemorrhage, 3) morphological factors of an aneurysm (daughter sac, irregular shape), and the potential modifications of the aneurysmal sac with time (increase in size, modification of the shape), and 4) patient preference. Once the decision to treat the aneurysm was made, endovascular treatment is preferred to surgery in the following cases: 1 ) a location with poor surgical accessibility, 2) clinical factors (age, medical condition), and 3) patient preference. From the view point of location only, it may be one of the most controversial issues (unruptured, 


\section{Endovascular Coil Embolization of Very Small Intracranial Aneurysms}

paraclinoid ICA aneurysm) that should be treated. Our study, to date, indicated that the results of the endovascular treatment of very small aneurysms performed under daily practice, and did not rationalize the indications of endovascular treatment for unruptured very small aneurysms.

There are various limitations to our study. Namely, untreated aneurysms were not registered and imaging follow-ups after embolization were performed using 3D TOF MRA; although most treatable aneurysm remnants can be visualized with reasonable confidence by using high-resolution TOF-MRA (18). The issue that unruputured very small aneurysms of paraclinoid ICA should be treated remains a matter of debate.

In conclusion, endovascular coil embolization of very small aneurysms was technically feasible and showed successful initial angiographic results without procedural morbidity and mortality, although two thromboembolic events occurred during the procedure not resulting neurologic deficits. On clinical and MRA follow-up, a good clinical outcome and no recurrence or bleeding of coiled aneurysms was noted. The long-term efficacy of coil embolization and its potential as a standard treatment strategy in very small aneurysms remains to be seen once randomized large trials are performed.

\section{References}

1. Molyneux AJ, Kerr RS, Yu LM, Clarke M, Sneade M, Yarnold JA, et al. International subarachnoid aneurysm trial (ISAT) of neurosurgical clipping versus endovascular coiling in 2143 patients with ruptured intracranial aneurysms: a randomised comparison of effects on survival, dependency, seizures, rebleeding, subgroups, and aneurysm occlusion. Lancet 2005;366:809-817

2. Nguyen TN, Raymond J, Guilbert F, Roy D, Bérubé MD, Mahmoud M, et al. Association of endovascular therapy of very small ruptured aneurysms with higher rates of procedure-related rupture. J Neurosurg 2008;108:1088-1092

3. Sluzewski M, Bosch JA, van Rooij WJ, Nijssen PC, Wijnalda D. Rupture of intracranial aneurysms during treatment with Guglielmi detachable coils: incidence, outcome, and risk factors. J Neurosurg 2001;94:238-240

4. Lim YC, Kim BM, Shin YS, Kim SY, Chung J. Structural limitations of currently available microcatheters and coils for endovascular coiling of very small aneurysms. Neuroradiology 2008;50:423-427

5. van Rooij WJ, Keeren GJ, Peluso JP, Sluzewski M. Clinical and angiographic results of coiling of 196 very small $(<$ or $=3 \mathrm{~mm})$ intracranial aneurysms. AJNR Am J Neuroradiol 2009;30:835839

6. Suzuki S, Kurata A, Ohmomo T, Sagiuchi T, Niki J, Yamada M, et al. Endovascular surgery for very small ruptured intracranial aneurysms. Technical note. J Neurosurg 2006;105:777-780

7. Pierot L, Spelle L, Vitry F; ATENA Investigators. Immediate clinical outcome of patients harboring unruptured intracranial aneurysms treated by endovascular approach: results of the ATENA study. Stroke 2008;39:2497-2504

8. Brinjikji W, Lanzino G, Cloft HJ, Rabinstein A, Kallmes DF. Endovascular treatment of very small (3 $\mathrm{mm}$ or smaller) intracranial aneurysms: report of a consecutive series and a meta-analysis. Stroke 2010;41:116-121

9. Cloft HJ, Kallmes DF. Cerebral aneurysm perforations complicating therapy with Guglielmi detachable coils: a meta-analysis. AJNR Am J Neuroradiol 2002;23:1706-1709

10. Moret J, Cognard C, Weill A, Castaings L, Rey A. Reconstruction technic in the treatment of wide-neck intracranial aneurysms. Long-term angiographic and clinical results. Apropos of 56 cases. J Neuroradiol 1997;24:30-44 [French]

11. Cognard C, Weill A, Spelle L, Piotin M, Castaings L, Rey A, et al. Long-term angiographic follow-up of 169 intracranial berry aneurysms occluded with detachable coils. Radiology 1999;212:348-356

12. Raymond J, Guilbert F, Weill A, Georganos SA, Juravsky L, Lambert A, et al. Long-term angiographic recurrences after selective endovascular treatment of aneurysms with detachable coils. Stroke 2003;34:1398-1403

13. Gallas S, Pasco A, Cottier JP, Gabrillargues J, Drouineau J, Cognard C, et al. A multicenter study of 705 ruptured intracranial aneurysms treated with Guglielmi detachable coils. AJNR Am J Neuroradiol 2005;26:1723-1731

14. Campi A, Ramzi N, Molyneux AJ, Summers PE, Kerr RS, Sneade M, et al. Retreatment of ruptured cerebral aneurysms in patients randomized by coiling or clipping in the International Subarachnoid Aneurysm Trial (ISAT). Stroke 2007;38:15381544

15. Murayama Y, Nien YL, Duckwiler G, Gobin YP, Jahan R, Frazee J, et al. Guglielmi detachable coil embolization of cerebral aneurysms: 11 years' experience. J Neurosurg 2003;98:959-966

16. Nguyen TN, Hoh BL, Amin-Hanjani S, Pryor JC, Ogilvy CS. Comparison of ruptured vs unruptured aneurysms in recanalization after coil embolization. Surg Neurol 2007:68:19-23

17. Wiebers DO, Whisnant JP, Huston J 3rd, Meissner I, Brown RD Jr, Piepgras DG, et al. Unruptured intracranial aneurysms: natural history, clinical outcome, and risks of surgical and endovascular treatment. Lancet 2003;362:103-110

18. Wallace RC, Karis JP, Partovi S, Fiorella D. Noninvasive imaging of treated cerebral aneurysms, Part I: MR angiographic follow-up of coiled aneurysms. AJNR Am J Neuroradiol 2007;28:1001-1008 